\title{
Aportes de los actores de la práctica pedagógica al desarrollo de las competencias específicas en la licenciatura en matemática en formación virtual y presencial ${ }^{*}$
}

\author{
Sonia Valbuena-Duarte ${ }^{* *}$ \\ María Angélica Jiménez Ávila*** \\ Wendy Jiménez Ávila*****
}

Recibido: 03-09-2020

Aceptado: 04-09-2020

\begin{abstract}
Citar como: Valbuena-Duarte, S., Jiménez Ávila, M. A. y Jiménez Ávila, W. (2021). Aportes de los actores de la práctica pedagógica al desarrollo de las competencias específicas en la licenciatura en matemática en formación virtual y presencial. Revista Interamericana de Investigación, Educación y Pedagogía, 14(2), 247-275. https://doi.org/10.15332/25005421.6114
\end{abstract}

\section{Resumen}

Esta investigación tiene como objetivo examinar el papel que desempeñaron los actores principales de la práctica pedagógica realizada por profesores en formación inicial en el desarrollo de las competencias específicas: enseñar, formar y evaluar del futuro profesor. Estas competencias son referentes en el área de educación, en pruebas estandarizadas para medir la calidad

\footnotetext{
* Este artículo es original y producto del proyecto de investigación realizado en la Licenciatura en Matemática de la Universidad del Atlántico, en el grupo de investigación GIMED.

** Magíster en Educación y en Matemáticas; doctoranda en Ciencias. Docente investigadora de la Licenciatura en Matemáticas y líder del grupo de investigación GIMED, categorizado en Colciencias, de la línea de investigación Formación de profesores de la Universidad del Atlántico, Colombia.

Correo electrónico: soniabalbuena@mail.uniatlantico.edu.co ORCID: https://orcid.org/0000-0003-3667-1087

Google Scholar: https://scholar.google.com/citations?user=ycC9egsAAAAJ\&hl=es

CvLAC: https://scienti.minciencias.gov.co/cvlac/visualizador/generarCurriculoCv.do?.cod_rh=0000428744

*** Licenciada en Matemáticas por la Universidad del Atlántico, Colombia. Miembro del grupo de investigación GIMED.

Correo electrónico: mariaangelicajimenez@mail.uniatlantico.edu.co

ORCID: https://orcid.org/0000-0003-0186-0857

**** Licenciada en Matemáticas por la Universidad del Atlántico, Colombia. Miembro del grupo de investigación GIMED.

Correo electrónico: wịoanajimenez@mail.uniatlantico.edu.co

ORCID: https://orcid.org/0000-0003-2886-2078
} 
de la formación del licenciado y el nivel de apropiación. Para ello se utilizó como metodología la revisión documental y entrevistas para recoger experiencias y percepciones de profesores de prácticas, profesores asesores y profesores en formación inicial de programas de Licenciatura en Matemática en modalidad virtual y presencial. Los resultados se centran en el papel de estos actores y su relación con los desarrollos de las competencias específicas del futuro profesor. Las conclusiones son el aporte al conocimiento de las características de espacios oportunos que propicien el desarrollo de las competencias específicas, además de la manera como puede contribuir cada actor.

Palabras clave: práctica pedagógica, competencia profesional, formación de docentes, educación a distancia virtual, enseñanza, formación, evaluación.

\title{
Contributions of the Actors of the Pedagogical Practice to the Development of Specific Competencies in the Mathematics Graduate in Virtual and Face-to-Face Training
}

\begin{abstract}
This research aims to examine the role played by the main actors of the pedagogical practice carried out by teachers in initial training in the development of the specific competencies: teaching, training, and evaluation. These competencies are benchmarks in the area of education in standardized tests to measure the quality of the graduate's training and the level of appropriation. For this purpose, a documentary review and interviews were used as a methodology to collect experiences and perceptions of internship teachers, advisory teachers, and teachers in initial training in the Bachelor of Mathematics in virtual and faceto-face modalities. The results found focus on the role of these
\end{abstract}


actors and their relationship with the development of specific competencies of the future teacher. The conclusions are the contribution to the identification of the characteristics of appropriate spaces that promote the development of specific competencies, as well as how each actor can contribute.

Keywords: pedagogical practice, professional competence, teachers training, virtual distance learning, teaching, training, evaluation.

\section{Contribuições dos atores envolvidos na prática pedagógica para o desenvolvimento das competências específicas do aluno para a licenciatura em matemática na formação virtual e presencial}

\section{Resumo}

Esta pesquisa teve como objetivo analisar o papel desempenhado pelos principais atores da prática pedagógica desempenhada por professores em formação inicial no desenvolvimento de competências específicas: ensinar, formar e avaliar o futuro professor. Essas competências são referências na área da educação em provas padronizadas para medir a qualidade da formação do graduado e o nível de apropriação. Para isso, a metodologia utilizada foi a revisão documental e entrevistas para coletar experiências e percepções de professores em estágio, professores orientadores e professores em formação inicial de programas de Bacharelado em Matemática na modalidade virtual e presencial. Os resultados encontrados enfocam o papel desses atores e sua relação com o desenvolvimento das competências específicas do futuro professor. As conclusões são a contribuição para o conhecimento das características de espaços oportunos que promovam o desenvolvimento de competências específicas, bem como a forma como cada ator pode contribuir. 
Palavras-chave: prática pedagógica, habilidades específicas do professor, professores em formação inicial, educação à distância, formação, avaliação.

\section{Introducción}

La optimización de la calidad educativa conlleva al mejoramiento de la formación del profesorado, lo que le implica al docente dejar atrás los modelos tradicionales de enseñanza y aprendizaje, y desarrollar competencias en los estudiantes, estimulando la lectura, la creatividad, el pensamiento crítico, entre otras habilidades necesarias para el ciudadano de este siglo. Sin embargo, para este logro es necesario impulsar el desarrollo profesional de los educadores (De Zubiría, 2019). En palabras de Andreucci (2013), Osorio, Herrera y Quiles (2016), se requieren formadores competentes.

En Colombia, el Instituto Colombiano para la Evaluación de la Educación (Icfes) evalúa la calidad de la educación superior (Artículo 7 de la Ley 1324 de 2009), para lo que ha creado un instrumento estandarizado conocido como las Pruebas Saber Pro (para el caso de educación terciaria). Cabe señalar que para el año 2010, el Icfes con apoyo de la Asociación Colombiana de Facultades de Educación (Ascofade) desarrollaron las especificaciones de los tres módulos de competencias específicas que conforman la prueba (Icfes, 2018a). Uno de los objetivos de las pruebas es verificar el desarrollo de competencias de los estudiantes que hayan cursado mínimamente el $75 \%$ de los créditos académicos de sus carreras.

La prueba está compuesta por subpruebas que evalúan competencias genéricas (de lectura crítica, razonamiento cuantitativo, competencias ciudadanas, comunicación escrita e inglés) y competencias específicas. Para el caso de los programas en educación estas son: enseñar, formar y evaluar.

Estas competencias específicas son campos primordiales en la estructura del saber profesional del profesor en formación o futuro docente (Icfes, 2018a). Asimismo, deben estar vinculadas 
al currículo de los programas que forman docentes, y se extienden en múltiples ámbitos de la práctica pedagógica, en la que participan, además del profesor en formación inicial, dos actores fundamentales: los profesores de prácticas pedagógicas de la universidad y los profesores que acompañan desde los centros de práctica al profesor en formación inicial.

Dada la trascendencia que tienen estas competencias, surge una alarma para los programas universitarios de formación inicial docente debido a los bajos desempeños obtenidos por los futuros profesores en las Pruebas Saber Pro. En el periodo de 2016 a 2019 en una escala de 0 a 300 puntos, el puntaje promedio obtenido ha sido inferior a 150.

Con el presente trabajo se busca contribuir en el estudio y conocimiento de la incidencia de los actores de la práctica pedagógica en el desarrollo y apropiación de las competencias específicas, teniendo en cuenta la responsabilidad de estos en las instituciones de educación superior y centros de prácticas. Estos últimos corresponden a instituciones educativas públicas o privadas, con las cuales las primeras realizan convenios para que los profesores en formación puedan llevar a cabo su práctica pedagógica.

Para esto se establece un marco conceptual sobre la importancia de las prácticas pedagógicas realizadas por el profesor de matemáticas en formación, así como de las competencias específicas de los docentes.

\section{Práctica pedagógica realizada por profesores en formación inicial}

La Ley 115 de 1994, Ley General de Educación, en su artículo 109, establece como parte primordial de la finalidad de la formación docente el saber desarrollar la teoría y la práctica pedagógica. Esta última será concebida en este trabajo, según lo establecido por el Ministerio de Educación Nacional (MEN), como un "proceso de autorreflexión, que se convierte en el espacio de conceptualización, 
investigación y experimentación didáctica, [...] en consecuencia, la práctica promueve el desarrollo de las competencias profesionales de los futuros licenciados" (MEN, 2016, p. 5).

Así las cosas, las prácticas pedagógicas se convierten para el estudiante, el profesor o el profesor en formación inicial, en la oportunidad para aproximarse al medio de vida profesional, en el que podrá aprender a resolver problemas y reconocer sus desempeños, además de interiorizar los conocimientos adquiridos. Por lo anterior, cobra sentido que estas prácticas sean el centro de los planes de estudio y se deban desarrollar de manera progresiva (Correa, 2014; Esquea, 2017; Fuentealba y Vanegas, 2019; Hernández, Quezada y Venegas, 2016; Parra, 2013). Colombia así lo ha asumido desde la normativa, a través de la Resolución 18583 de 2017, en la cual también se establece que las prácticas deben desarrollarse por lo menos en 40 créditos académicos presenciales. Tomando como promedio aproximado 160 créditos totales de las carreras de licenciaturas, la práctica está representando por lo menos el $25 \%$ de los créditos del plan de estudios, lo que muestra el lugar importante que tiene en este.

\section{Competencias específicas}

Para Tobón (2006, p. 1) "las competencias son un enfoque para la educación y no un modelo pedagógico", así el concepto de competencia más difundido en el ámbito educativo es "saber hacer en situaciones concretas que requieren la aplicación creativa, flexible y responsable de conocimientos, habilidades y actitudes" (MEN, 2006, p. 12). Por lo tanto, el concepto de competencia no se limita a las competencias laborales (Tuning América Latina, 2007), sino que involucra el saber, el saber hacer, las aptitudes, el saber ser, las actitudes y los valores. Por lo anterior, es posible que se desarrollen mediante procesos en un contexto específico y cambiante, que conlleve al sujeto responsable a ser competente. 
En consecuencia, se reconocen diferentes tipos de competencias, dentro de las cuales se encuentran las competencias específicas (Icfes, 2018a), las cuales son particulares, básicas y fundamentales del saber de los educadores. Estas incluyen componentes teóricos y prácticos en los campos de enseñar, evaluar y formar. Es así que estas tres competencias tienen relación directa con el campo de estudio y con las habilidades y capacidades de los futuros docentes.

Por otra parte, evaluar las competencias profesionales a nivel superior es posible en el prácticum (Tejada y Ruiz, 2016) o en trabajos de fin de grado. En la literatura, los términos práctica profesional, práctica académica y prácticum se refieren al momento en que ocurre la práctica pedagógica (Bolívar, 2019). Para el caso de los programas de formación inicial docente, la práctica pedagógica constituye el espacio donde se despliegan las competencias específicas: enseñar, evaluar y formar (Icfes, 2018a).

\section{Metodología}

El objetivo del presente artículo es analizar el aporte de los actores del proceso de las prácticas pedagógicas en el desarrollo de las competencias específicas en los profesores en formación de programas de licenciatura. El diseño metodológico utilizado es un estudio de casos múltiple (Stake, 2006), y la metodología se organizó en cuatro fases adaptadas de Moreno (2015): la primera fase de exploración documental; la segunda de trabajo de campo; como tercera fase se realizó la organización y clasificación de la información; y la última fase fue de análisis y sistematización de la información.

\section{Participantes}

Con un muestreo intencional (Vasilachis, et ál., 2006) se conformó el grupo de participantes de la muestra, el cual estaba constituido por los actores del proceso de práctica: coordinadores de práctica, 
profesores de práctica (PP), profesores asesores (PA) y profesores en formación (PF) inicial. Todos los participantes pertenecían a dos programas de licenciatura en matemáticas de dos universidades de carácter oficial; uno de los programas es ofrecido en modalidad presencial en la Región Caribe colombiana y el otro programa es ofertado en modalidad a distancia virtual en una universidad que tiene presencia en todo el territorio colombiano. El PP es el profesor encargado de la asignatura de prácticas pedagógicas en la universidad. Por su parte, el docente de aula de la institución educativa en donde el PF desarrolla sus prácticas pedagógicas será identificado como profesor asesor (PA).

Se seleccionaron dos coordinadores de práctica, uno del programa de Licenciatura en Matemáticas en modalidad presencial y otro del programa de Licenciatura en Matemáticas en modalidad virtual; cinco PP, de los cuales cuatro son del programa en modalidad presencial y uno del programa de modalidad virtual; cinco PA; y cinco PF de cada programa. Los PF son estudiantes de último año de sus carreras de Licenciatura en Matemáticas, que hayan realizado o estén cursando la práctica pedagógica.

\section{Técnicas e instrumentos para la recolección de información}

En el trabajo de campo se recolectaron datos verbales y escritos a través de entrevistas aplicando cuestionarios a la muestra seleccionada. Esto con el objetivo de indagar, para el caso de los coordinadores de prácticas, sobre la estructura formal del proceso de prácticas y cómo este es Ilevado a cabo. En el cuestionario aplicado a los PP y a los PA, se preguntaba acerca de sus propias percepciones sobre el papel que desempeñan en el proceso de prácticas y las competencias que pretendían desarrollar en los PF. Un tercer cuestionario fue aplicado a los profesores en formación, con el objetivo de indagar acerca de los aprendizajes y competencias adquiridas durante el proceso de prácticas pedagógicas.

Los reglamentos de prácticas y los sílabos de prácticas de los dos programas académicos constituyeron fuentes de información 
en la búsqueda de los aportes que, desde esta parte documental, pudieran hacer los profesores actores de la práctica al proceso de desarrollo de las competencias específicas del profesor en formación inicial.

Los puntajes obtenidos por los estudiantes de la muestra objeto de estudio en las competencias específicas de las Pruebas Saber Pro son una tercera fuente. Esto con el propósito de obtener información del desarrollo y perfeccionamiento de estas competencias, teniendo como base que la Prueba evalúa cómo emplear dichos conceptos para resolver problemas en situaciones de la vida cotidiana del área profesional (Icfes, 2019).

\section{Técnica de análisis de la información}

En la fase de organización y clasificación, la información recolectada con las entrevistas se hizo por actor y por modalidad del programa académico. Para el análisis de los datos se utilizó la triangulación de la información obtenida en las entrevistas, el análisis didáctico y los aportes teóricos consultados durante la exploración documental.

Para analizar los aportes que hace la práctica pedagógica al desarrollo de las competencias específicas del profesor en formación desde lo documentado en los reglamentos de práctica y los sílabos de estos cursos, se aplicó la técnica del análisis didáctico a estos documentos, siguiendo los lineamientos de Rico (2013). Estos resultados se estructuraron en cinco análisis: conceptual, de contenido, cognitivo, de instrucción y de evaluación.

También se llevó a cabo un análisis de tipo descriptivo, al presentar mediante gráficas los resultados obtenidos por los estudiantes de los dos programas en las competencias específicas evaluadas por el Icfes en los años 2016, 2017 y 2019. Para este análisis se utilizó la conceptualización de cada competencia en términos de sus descriptores o afirmaciones $(A)$, así:

Enseñar: comprende el uso de la didáctica de las disciplinas en la enseñanza (A1), diseña proyectos curriculares, planes 
de estudio y unidades de aprendizaje (A2) y promueve actividades de enseñanza y aprendizaje que favorezcan el desarrollo conceptual y procedimental de los estudiantes (A3). Evaluar: comprende los aspectos relacionados con: que el estudiante conozca diversas alternativas para evaluar (A1), comprenda el impacto de la evaluación para el mejoramiento de los procesos educativos (A2) y además comprenda la relevancia de la autorregulación en los sujetos de la educación (A3). Formar: Comprende las características físicas, intelectuales y sociales de sus estudiantes (A1), entiende la importancia del desarrollo cultural de sus estudiantes (A2), comprende sus propios procesos de desarrollo profesional y busca mejoramiento continuo (A3), vincula sus prácticas educativas con el reconocimiento de la institución educativa como centro de desarrollo social y cultural (A4). (Icfes, 2019, pp 9-10)

\section{Resultados}

Para analizar los aportes que hace la práctica pedagógica al desarrollo de las competencias específicas del profesor en formación inicial, desde lo documentado en los reglamentos de práctica y los sílabos de estos cursos, en este apartado se presentan los resultados obtenidos al aplicar la técnica del análisis didáctico, siguiendo los lineamientos de Rico (2013). A continuación, se describen los resultados para cada ciclo de análisis.

\section{Análisis conceptual}

En los reglamentos de prácticas de los programas analizados en modalidad presencial y virtual — que se materializa en los sílabos-, la práctica pedagógica se conceptualiza como un espacio de formación, reflexión e investigación, en donde el estudiante demuestra las competencias adquiridas a lo largo de toda la carrera, y desarrolla competencias profesionales. Sin embargo, también se revela 
la ausencia de aspectos importantes, tales como la autorreflexión, la innovación y la experimentación didáctica (MEN, 2016), además de un atributo con alto valor agregado que debe dar la práctica pedagógica: formar la identidad del futuro profesor (Correa, 2014; Fuentealba y Vanegas, 2019). En todos los hallazgos no es evidente el papel que desempeñarían los PP y PA en este sentido.

\section{Análisis de contenido}

En la estructura de los reglamentos de práctica de los programas presencial y virtual se contemplan diferentes niveles o fases para el desarrollo de la práctica pedagógica: un primer momento de observación, luego de ayudantías y, finalmente, las prácticas profesionales o de inmersión (MEN, 2016); así se estructuran los microcurrículos para cada uno de estos cursos.

\section{Análisis cognitivo}

Al analizar las expectativas de aprendizaje en los reglamentos de prácticas del programa en modalidad presencial, se espera que el PF desarrolle competencias profesionales pedagógicas tales como la gestión, el diseño y el desarrollo didáctico de proyectos pedagógicos institucionales. Además, se busca que lleve a cabo actividades de enseñanza y aprendizaje, en las cuales la articulación de conceptos y procedimientos de los saberes de la disciplina, la didáctica, la historia, la epistemología y la pedagogía, constituyan su fundamento.

De manera más simple, en el reglamento de prácticas del programa en modalidad virtual, se espera que el PF refuerce procesos pedagógicos, didácticos, tecnológicos e investigativos que conduzcan al reconocimiento y compromiso frente a su quehacer como maestro en formación. Sin embargo, es de anotar que en los sílabos no se halló evidencia de cómo cada actor de la práctica materializaría estas expectativas de aprendizaje. 


\section{Análisis de instrucción}

En la organización y gestión del trabajo en el aula se analiza el papel que desempeñan los diferentes actores del proceso de prácticas pedagógicas, como lo son: coordinador de prácticas, los profesores PP, PA y PF.

El coordinador de prácticas solo tiene funciones relacionadas con los aspectos legales y administrativos de las prácticas. Por su parte y de manera general en los programas presenciales y virtuales, para el profesor de prácticas (PP) se identifica una función marcada de seguimiento al proceso de las prácticas pedagógicas. Esto en cuanto al cumplimiento de aspectos legales y administrativos, como el manejo de formatos de inscripciones, reportes de asistencia a los centros de práctica y calificaciones del PF, y la selección de los centros de práctica, así como dirigir y orientar al PF en las actividades propias de la práctica y sus funciones.

En los reglamentos de práctica, el profesor asesor (PA) acompaña al PF al interior del centro de prácticas y emite un juicio de valor en la evaluación de su práctica. El formato de evaluación es diligenciado con base en la observación y análisis de los desempeños académico, pedagógico y administrativo del PF. Se espera que sea el encargado de orientarle en los procesos relacionados con la pedagogía y la didáctica. Cabe destacar que en el sílabo no se evidencia de qué forma lo haría, es decir, no se indica la estrategia que pudiera seguirse.

Para el PF, los reglamentos de prácticas pedagógicas le establecen criterios de comportamientos que debe tener en los centros de práctica y con la comunidad educativa de estos. Además, señala aspectos normativos relacionados con el cumplimiento de horarios y actividades encomendadas, la lectura del reglamento de prácticas, el alistamiento de la documentación requerida al iniciar y terminar el proceso de prácticas pedagógicas, y el cumplimiento a las citaciones por parte del PP. 
En los programas analizados se identifican papeles y funciones de los actores principales de la práctica pedagógica con un marcado acento a lo administrativo y a la gestión de la práctica. Sin embargo, no se evidencian aspectos relacionados con las competencias necesarias para asumir dicho papel.

\section{Análisis de evaluación}

En los reglamentos de práctica se establece una evaluación a partir del desarrollo de competencias disciplinares, cognitivas, pedagógicas, actitudinales, administrativas, conciliatorias, comunicativas e investigativas. Los sílabos de los programas académicos presenciales y virtuales analizados contemplan en este aspecto la gestión de formatos, sin un proceso de retroalimentación y reflexión para el PF. Con ello se deja de lado un momento importante en la producción de conocimiento, como se destaca en investigaciones previas (Sánchez, 2016; Llinares, Ivars, Buforn y Groenwald, 2019).

\section{Análisis de los resultados en las competencias específicas evaluadas por el lcfes}

En Colombia y el mundo el indicador más usado para medir el logro académico son las pruebas estandarizadas (Ahumada, Gamboa y Guerrero, 2018). A nivel nacional, el Icfes es el encargado de elaborar y aplicar instrumentos de evaluación de la calidad de la educación a estudiantes de básica, media y superior, según las orientaciones que para ello defina el MEN (Icfes, 2019).

A continuación, se presentan los resultados obtenidos por los PF de los programas académicos analizados en las competencias específicas (enseñar, evaluar y formar) de la Prueba Saber Pro aplicada por el Icfes durante los años 2016, 2017 y 2019. Dichos resultados se analizan pretendiendo una visión más amplia acerca 
del desarrollo y perfeccionamiento de estas competencias en los PF. Además, se relacionan estos desarrollos con los aportes que reciben en didáctica, pedagogía y en el saber hacer desde la práctica pedagógica.

\section{Competencia de estudio "enseñar" presentada por afirmaciones}

La figura 1 presenta el porcentaje de respuestas erradas por afirmación. En la línea de tiempo analizada, en general, lo recurrente ha sido que los estudiantes de las dos modalidades tienen más del $40 \%$ de respuestas erradas en los descriptores de la competencia, a excepción de la modalidad virtual para el año 2017, que obtuvo menos del $40 \%$ en los descriptores A1 y A2. Hubo una mejoría gradual representada en la disminución en el porcentaje de respuestas erradas en los años 2016, 2017 y 2019 en cuanto A3.

Figura 1. Porcentaje de respuestas erradas de los PF de los dos programas en estudio en la competencia enseñar.

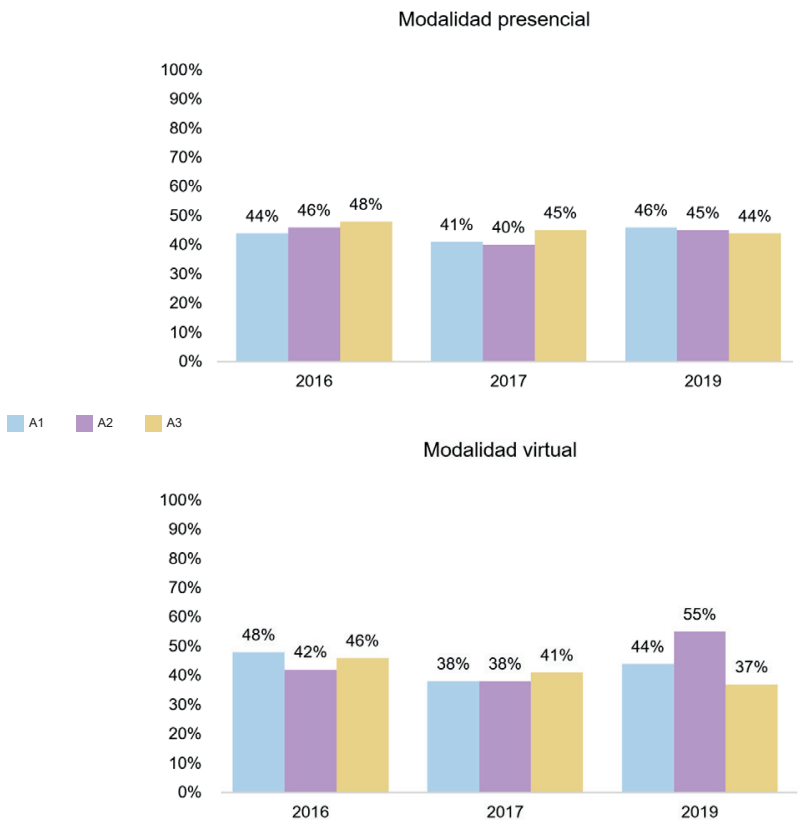

Fuente: elaboración con base en los datos del Icfes (2018b, 2019). 
Estos resultados muestran bajos desarrollos de los PF de las dos modalidades en el uso de la didáctica para favorecer los aprendizajes de los estudiantes. Este indicador fue más acentuado en el caso del programa en modalidad presencial.

\section{Competencia de estudio "evaluar" presentada por afirmaciones}

En la figura 2 se observa bajos desempeños del PF en la competencia, con una ligera mejoría en el último año en las habilidades A1 y A2. Sin embargo, en el programa presencial se evidencia poca comprensión de la relevancia de la autorregulación (A3), puesto que, en todos los años analizados, los estudiantes obtuvieron un porcentaje de preguntas erradas superior al $50 \%$.

Figura 2. Porcentaje de respuestas erradas en la competencia evaluar obtenido por los PF de los programas analizados.

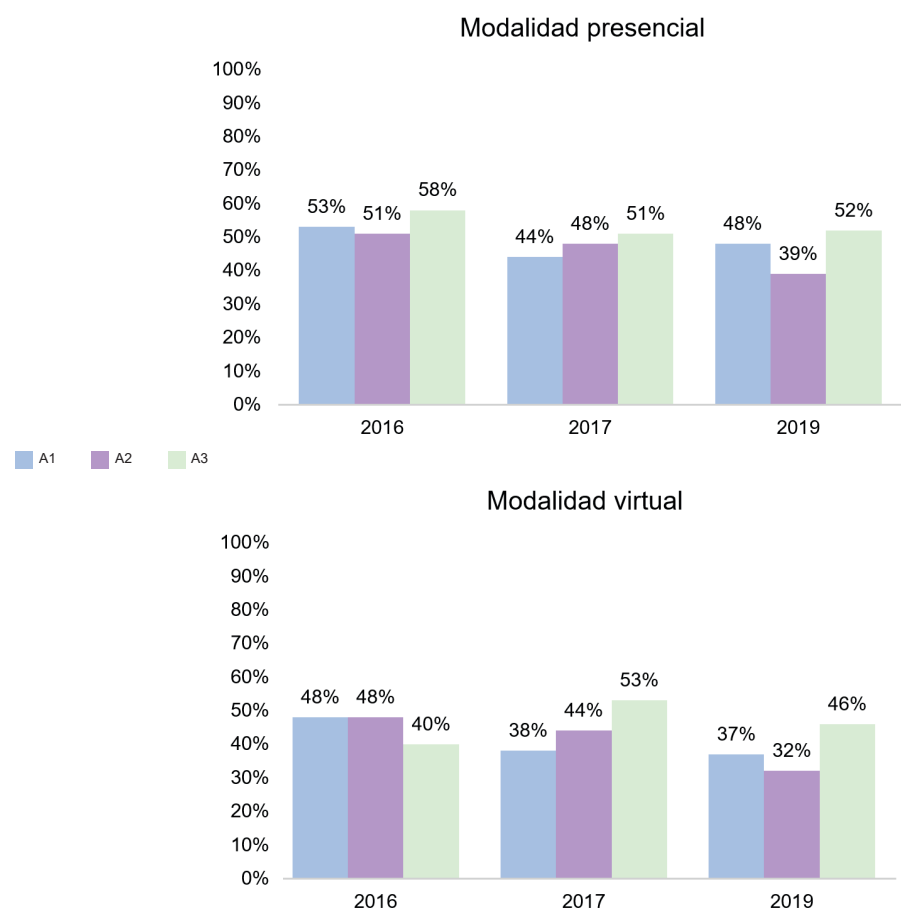

Fuente: elaboración con base en los datos del Icfes (2018b, 2019). 


\section{Competencia de estudio "formar" presentada por afirmaciones}

Con respecto a esta competencia, en la figura 3 se evidencian resultados similares a la de evaluar. En las afirmaciones $A 1$ y A2, los PF del programa en modalidad presencial en todos los años analizados obtuvieron un porcentaje de respuestas erradas superior al $50 \%$, mientras que los PF del programa en modalidad virtual obtuvieron un porcentaje de respuestas erradas inferior al $50 \%$.

Figura 3. Porcentaje de respuestas erradas en la competencia formar obtenido por los futuros profesores del programa en modalidad presencial y del programa en modalidad virtual

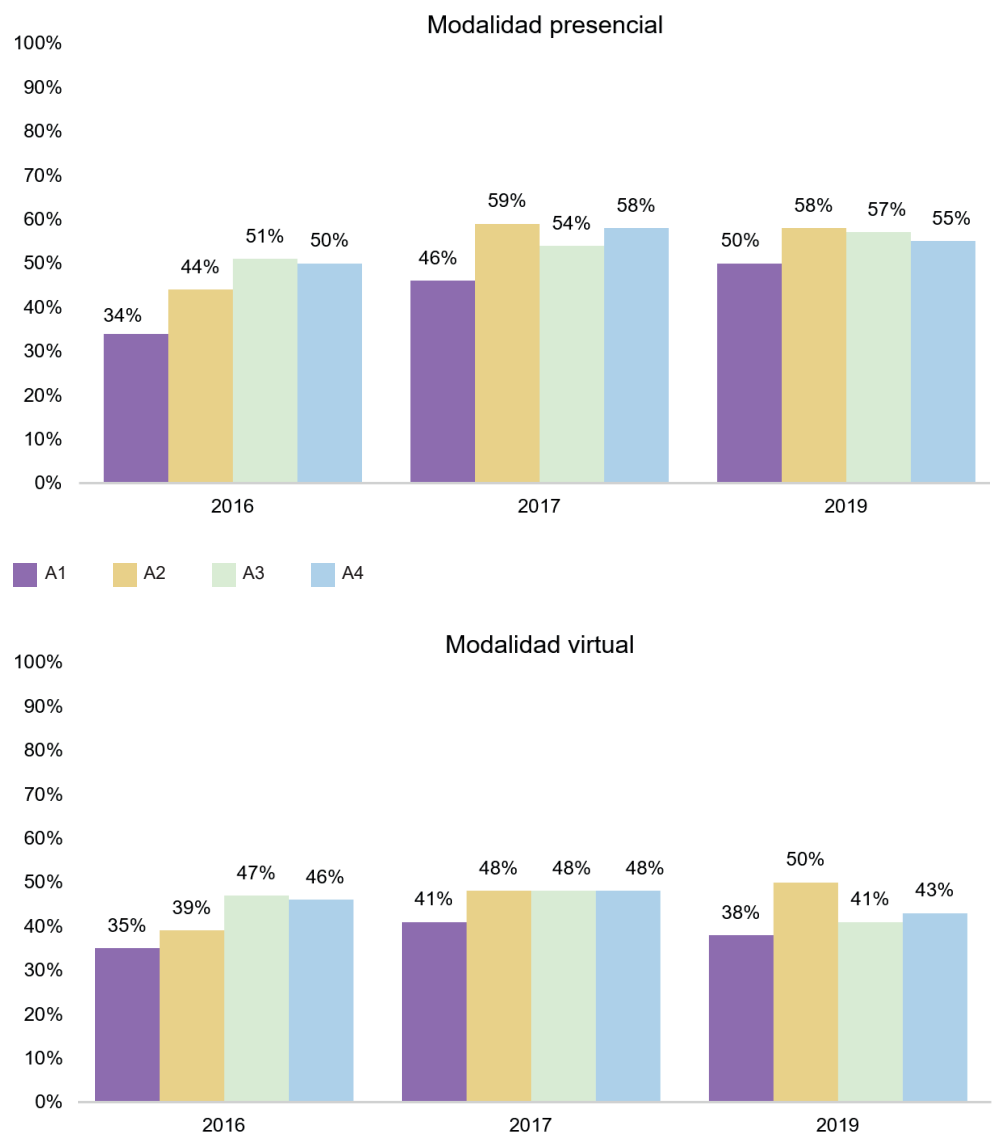

Fuente: elaboración con base en los datos del Icfes (2018b, 2019). 
A manera de comparación de los desempeños en las competencias específicas de los PF de los programas objeto estudio con otros programas nacionales de licenciaturas, en la tabla 1 se muestra el porcentaje de respuestas erradas obtenido por los estudiantes de cuatro programas académicos (dos en modalidad presencial y dos en modalidad virtual) para la competencia enseñar. Se evidencia que los resultados son similares en los programas estudiados, con eventuales mejorías en los programas presenciales en algunas habilidades (A1 y A3 en 2019 y A2 en 2017). Esto sucede en la mayoría de las afirmaciones de las competencias evaluar y formar, en las que los estudiantes obtuvieron un porcentaje de respuestas erradas superior al $40 \%$.

Tabla 1. Porcentaje de respuestas erradas en la competencia enseñar obtenido por los estudiantes en modalidad presencial y virtual de cuatro programas

\begin{tabular}{|c|c|c|c|c|c|c|c|c|c|}
\hline \multirow{2}{*}{ Nivel de agregación } & \multicolumn{3}{|c|}{ Afirmación } \\
\cline { 2 - 9 } & \multicolumn{3}{|c|}{ A1 } & \multicolumn{3}{|c|}{ A2 } & \multicolumn{3}{c|}{ A3 } \\
\hline Ensenar & 2016 & 2017 & 2019 & 2016 & 2017 & 2019 & 2016 & 2017 & 2019 \\
\hline Programa presencial 1 & $54 \%$ & $46 \%$ & $33 \%$ & $46 \%$ & $31 \%$ & $50 \%$ & $41 \%$ & $43 \%$ & $21 \%$ \\
\hline Programa virtual 1 & $54 \%$ & $49 \%$ & $50 \%$ & $46 \%$ & $46 \%$ & $61 \%$ & $49 \%$ & $53 \%$ & $45 \%$ \\
\hline Programa presencial 2 & $52 \%$ & $39 \%$ & $49 \%$ & $47 \%$ & $39 \%$ & $56 \%$ & $51 \%$ & $48 \%$ & $42 \%$ \\
\hline Programa virtual 2 & $49 \%$ & $42 \%$ & $48 \%$ & $44 \%$ & $46 \%$ & $57 \%$ & $51 \%$ & $48 \%$ & $44 \%$ \\
\hline Grupo de referencia NBC & $52 \%$ & $44 \%$ & $46 \%$ & $47 \%$ & $43 \%$ & $54 \%$ & $50 \%$ & $48 \%$ & $42 \%$ \\
\hline
\end{tabular}

Fuente: elaboración con base en los datos del Icfes (2018b, 2019).

Los resultados son un indicador de los desempeños de los PF al ejecutar acciones con cierta complejidad, que entrelazan procesos de pensamiento. Causa preocupación que los resultados obtenidos en los dos programas analizados estén lejos del ideal, que según el Icfes (2019) es de un porcentaje de respuestas erradas inferior al $20 \%$. Esto permite inferir que los programas requieren incrementar esfuerzos para aumentar la capacidad de los PF en relación con cada una de las competencias específicas (enseñar, evaluar y formar), puesto que ellas son base insustituible de la formación del futuro profesor y se requiere que las desarrolle e inicie su perfeccionamiento en su estancia de formación. 
Esta inferencia va en consonancia con el énfasis que, desde el 2002, se hace a América Latina sobre la necesidad de incrementar esfuerzos para el mejoramiento de la formación inicial docente. Aquí, la evaluación es un indicador de esta necesidad; no solo la evaluación escrita — que utilizan países como Colombia, Bolivia, México y Brasil-, sino también la evaluación de la práctica de investigación y observación en el aula (Schulmeyer, 2004), en lo cual participan el PP y el PA.

En Chile se evalúa a los profesores en formación inicial a través de la prueba Inicia, la cual se orienta a objetivar las competencias docentes que permitan retroalimentar procesos y estrategias formativas individuales e institucionales, para mejorar la calidad de la formación inicial docente. Los resultados de la prueba para el 2012 indicaron que en el área de ciencias (matemática, biología, física y química) no se lograron los estándares de calidad al evaluar el conocimiento pedagógico y disciplinar (Ruffinelli, 2013; Pino, Vargas, Larraín y Vargas, 2018). Esto coincide con los resultados nacionales.

La Organización de las Naciones Unidas para la Educación, Ciencia y la Cultura. (Unesco) considera cinco aspectos críticos de la formación docente en el contexto de la formación en América Latina y el Caribe, dentro de los cuales incluye la preparación del formador de formadores (Unesco, 2013). Lo anterior refuerza este trabajo investigativo, dada la relevancia del PP y el PA en el desarrollo de las competencias específicas del PF.

Para el estudio realizado, del programa presencial se destacan las relacionadas con la planeación de la clase, diseño curricular, liderazgo, responsabilidad, puntualidad, orden, y las competencias comunicativas, pedagógicas y disciplinares. Estas últimas se asocian con la competencia enseñar. Llama la atención que los programas virtuales muestran mayor énfasis en la competencia formar, y dan mayor relevancia y peso al comprender el aprendizaje de las matemáticas, el fortalecimiento de las habilidades de pensamiento, las causas y problemáticas de la enseñanza. Cabe resaltar que en ambos casos no se destaca la competencia evaluar, por lo cual no se pudo apreciar el seguimiento a los procesos ni la utilización 
de los resultados para mejorar el currículo, o el reconocimiento de la evaluación como elemento que restablece la calidad de este.

En ninguno de los programas tampoco se encontró el componente de reflexión, incluido en los dominios de la competencia enseñar. Este componente es considerado por la muestra seleccionada como el relato de las experiencias o sucesos que el PF presenció o vivenció en los centros de prácticas y los consejos o comentarios recibidos por el PP y el PA. Esto coincide con investigaciones previas (Fuentealba y Vanegas, 2019) en cuanto al poco carácter autorreflexivo dado a la práctica en los contextos reales de su desarrollo.

Este carácter autorreflexivo contribuye al entendimiento de la complejidad del proceso educativo (Font, 2011), el cual se facilita en la práctica pedagógica, donde se pueden lograr procesos cognitivos superiores, que encaminen a la reflexión sobre la práctica pedagógica (Font y Morales, 2017). Es precisamente en estos escenarios en los que el PP y el PA pueden generar espacios de diálogo entre todos los actores del proceso, para el logro de una práctica reflexiva.

De las entrevistas realizadas a los PF, un hallazgo por resaltar — sin importar la modalidad de estudio del programa académicoes que de manera general se relatan pocas contribuciones significativas del PA al desarrollo de las competencias específicas. Estos manifiestan que existen pocos encuentros para dialogar sobre sus desempeños en la práctica y los encuentros se reducen, en gran medida, a la recolección de firmas en los formatos de asistencias al centro de práctica y en unos pocos casos a las firmas y revisiones de sus planes de clase.

Las contribuciones del PP al desarrollo de las competencias enseñar, formar y evaluar — según la visión del PF- son en las competencias comunicativas, pedagógicas y reflexivas; también en aspectos como la puntualidad y el incentivo a la lectura sobre diversas formas de enseñar y aprender. 
De estos resultados se destaca que los actores principales de la práctica identifican la formación y el desarrollo de competencias; sin embargo, en este trabajo se agrega que estas no se relacionan en su totalidad con las competencias específicas de enseñar, formar y evaluar. Esto puede estar asociado con la ruptura entre la tríada formativa, al darse una comunicación por pares; es decir PPPF, PA-PF y, escasamente, PP-PA solo para aspectos de evaluación al PF. Esto puede deberse al notorio desconocimiento por parte de estos actores de la conceptualización y pretensión de las competencias específicas, y por la falta de claridad del papel asumido en el desarrollo de estas por el PF.

\section{Conclusiones}

Por su naturaleza, se reconoce que la práctica pedagógica aporta al desarrollo y consolidación de las competencias específicas del profesor en formación inicial. Por ello y a partir de una revisión profunda en los programas de licenciaturas en matemática analizados, se hace necesario retroalimentar y hacer procesos continuos de autoevaluación y planes de mejoramiento de las acciones de cada actor principal de la práctica y su aporte al desarrollo de estas competencias.

Esto constituye una contribución en la búsqueda de atenuar las debilidades en la formación del profesor (Osorio et ál., 2016), pues se reconoce que una docencia de calidad es fundamental para lograr un mejor aprendizaje en el estudiante. Para Pedró (2020), esto constituye una variable más influyente en el rendimiento escolar que otros factores como, por ejemplo, el tamaño de la clase.

De la revisión documental realizada, el análisis de las entrevistas aplicadas, la relación establecida entre lo considerado por los autores y lo encontrado en los reglamentos de prácticas y en los sílabos, y lo manifestado en las entrevistas de los programas académicos, se logró evidenciar que las competencias específicas enseñar, evaluar y formar - consideradas como básicas y fundamentales del saber 
profesional docente- están relacionadas con las descritas en los reglamentos de prácticas de los programas de Licenciatura en Matemáticas analizados; sin embargo, no se asocian con los sílabos ni con las consideradas por los actores principales de la muestra. Esto último puede generar que, desde la práctica pedagógica, no se procure el desarrollo de las competencias específicas en los profesores en formación inicial.

En los programas académicos — sin importar la modalidad de desarrollo-, a pesar de que la conceptualización de los actores de la práctica no se relaciona directamente con el desarrollo de las competencias específicas, se manifestaron otras del tipo comunicativo, actitudinal y administrativo, desarrolladas por parte del profesor en formación. Sin embargo, se hace necesario que desde los reglamentos de prácticas exista una clara concordancia entre las competencias que se desean desarrollar en el profesor en formación durante la práctica pedagógica y aquellas básicas y fundamentales que este debe desarrollar y perfeccionar. Además, se debe especificar los papeles y las responsabilidades del profesor de prácticas pedagógicas y el profesor asesor en el desarrollo de estas competencias en el profesor en formación, así como la responsabilidad que los centros de prácticas asumen al recibir formadores en un proceso de prácticas pedagógicas.

Los resultados conllevan a repensar los procesos y a generar acciones desde la práctica pedagógica que promuevan el desarrollo y apropiación de las competencias específicas. Esto con el propósito de fortalecer la relación de transferencia de saberes entre los actores de la práctica. Esto supondría la conformación de comunidades de práctica, como lo reportado en investigaciones anteriores (Barrios-Martínez et ál., 2019).

Este estudio contribuye al conocimiento de las características de espacios oportunos que propicien el desarrollo de las competencias específicas en el PF. Asimismo, se encuentra necesario y deseable que formadores (PP y PA) también desarrollen competencias que los acrediten en el campo de estudio, tales como hábitos investigativos, capacidad de análisis de su propia práctica, 
innovación y formación continua en el área educativa. Este aspecto ha sido destacado en otras investigaciones (Espinoza, 2018; Rivero, Carménate y León, 2019; Silva, 2010).

En cuanto a la caracterización de espacios para el desarrollo y perfeccionamiento de las competencias específicas del PF, otra contribución es la necesidad de competencias tecnológicas en los actores formadores (PP y PA) para el uso pedagógico y didáctico de la tecnología, pues es claro que esta ha transformado totalmente la educación (Acevedo, Pinto y Lemos, 2020; Glasserma, Rubio y Ramírez, 2013; Grajales y Osorio, 2019; Llinares et ál., 2019). Por lo tanto, se le atribuye a los PP y los PA crear los espacios y momentos para que el PF no se limite a una visión trivial de la enseñanza y el aprendizaje (competencia específica enseñar). Es claro que estos requerimientos agregan mayor exigencia al perfil de los formadores (Correa, 2014; Hernández et ál., 2016) y complejizan su tarea de educar.

Con la evaluación de la práctica pedagógica es deseable el desarrollo de una cultura de autorregulación (competencia específica evaluar) que incentive su transformación (Benítez, 2016) y le permita al PF desarrollar destrezas profesionales, identificar sus potenciales como profesor, planificar el perfeccionamiento y desarrollo profesional individual y colectivo, identificar y aprender a autoevaluarse, y elaborar planes de mejoramiento continuo (competencias específicas evaluar y formar) de los desempeños en sus competencias.

En cuanto al aporte del PA como actor interviniente en la práctica, se identifica la necesidad de dar el reconocimiento necesario a su papel para el éxito del desarrollo de las competencias específicas en el PF, indistintamente de la modalidad en que se oferte el programa académico. De igual manera, este actor le acerca al uso cotidiano de los medios que tienen los estudiantes para aprender, y le debería incentivar para diseñar y moderar entornos de aprendizaje virtuales y no virtuales (competencia específica enseñar). Asimismo, le pone en contacto con las llamadas comunidades de aprendizaje (Eirín-Nemiña, 2018), en las que se comparte y 
construye conocimiento; estos escenarios son determinantes para fomentar la investigación y la enseñanza (competencia específica formar). Sin embargo, es claro que para que este papel impacte como se espera en el desarrollo de las competencias específicas del PF, debe estar implicada la parte administrativa del centro de práctica.

Se concluye que, indistintamente de la modalidad en que se oferte un programa académico de licenciatura en matemáticas, se espera que el papel de los formadores apunte al mismo objetivo en relación con el desarrollo y perfeccionamiento de las competencias de enseñar, formar y evaluar del futuro profesor. Los procesos de globalización han abierto la posibilidad a diversos escenarios para acceder a formación de orden superior de manera especial a personas de bajos recursos (González, Berdugo y Mortigo, 2017), que no tienen la posibilidad de desplazarse a centros de educación superior desde lugares geográficamente remotos. Esto le ha dado un impulso a la educación a distancia; sin embargo, se han generado controversias en relación con la calidad de los profesionales que se forman en esta modalidad en comparación con la formación presencial.

Por lo anterior, se destaca que, a partir de los análisis realizados en este trabajo investigativo en cuanto a los resultados de las Pruebas Saber Pro - tomando este referente como medida de calidad (González et ál., 2017) — de los futuros profesores de matemática formados en la modalidad virtual o presencial, en cuanto a los desarrollos en las competencias específicas son claramente comparables y con algunos desempeños superiores en la primera de las modalidades (virtual). En otros trabajos (Cifuentes, Chácon y Fonseca, 2020) se ha llegado a conclusiones similares para el caso de las competencias genéricas del futuro profesor.

En síntesis, en cuanto a los roles del profesor de prácticas y del profesor asesor, la atención a estos actores del proceso tiene una relación importante e influyente con el desarrollo de las competencias específicas en el profesor en formación inicial, por lo que requiere mayor atención. Este estudio fue realizado con dos programas 
de licenciatura en Matemáticas; sin embargo, pueden generalizarse para todo programa que forme a licenciados o profesionales en educación, en donde la práctica pedagógica está presente como elemento importante en la formación.

\section{Referencias}

Acevedo, S., Pinto, D. y Lemos, A. (2020). Mediación pedagogica en la narrativa visual de cursos virtuales introductorios a licenciaturas. Revista Interamericana de Investigación, Educación y Pedagogía (RIIEP), 13(1), 113-136.

Ahumada, V., Gamboa, M. y Guerrero, J. H. (2018). Calidad de la Educación Superior en Colombia: Eficacia de algunos programas académicos presenciales y a distancia en las pruebas Saber Pro. UNAD Sello Editorial.

Andreucci, P. (2013). La supervisión de prácticas docentes: una deuda pendiente de la formación inicial de profesores. Estudios Pedagógicos; 39, 7-26.

Barrios-Martínez, D., Zuluaga-Ocampo, Z., García-Cepero, M., GómezHernández, F., Santamaría, A., Castro-Fajardo, L. y Sánchez-Vallejo A. (2019). Comunidades de práctica como marco comprensivo del talento docente. Magis, Revista Internacional de Investigación en Educación, 11(23), 75-94. https://doi.org/10.11144/Javeriana.m11-23.cpmc

Benítez, L. (2016). Evaluación e intervención pedagógica en la formación de docentes. Una acción reflexiva en el aula de clases. Revista de Investigación Educativa de la REDIECH, 7(12), 42-51. http://dx.doi. org/10.33010/ie_rie_rediech.v7i12

Bolívar, R. (2019). Investigar la práctica pedagógica en la formación inicial de maestros. Apertura, (51), 9-22. https://doi.org/10.17227/pys. num51-2881

Cifuentes, J., Chácon, J. y Fonseca, L. (2020). Análisis de los resultados de las pruebas saber pro en los estudiantes de la licenciatura en Educación Básica de la Universidad Pedagógica y Tecnólogica de Colombia (UPTC). Plumilla Educativa, 25(1), 125-151. http://dx.doi.org/10.30554/pe.1.38 33.2020 
Congreso de la República de Colombia. (1994, 8 de febrero). Ley 115 de 1994. Por la cual se expide la Ley General de Educación. Diario oficial 41 214.

Congreso de la República de Colombia. (2009, 13 de julio). Ley 1324 de 2009. Por la cual se fijan parámetros y criterios para organizar el sistema de evaluación de resultados de la calidad de la educación, se dictan normas para el fomento de una cultura de la evaluación, en procura de facilitar la inspección y vigilancia del Estado y se transforma el ICFES. Diario oficial 47409.

Correa, E. (2014). Las prácticas: primer espacio de profesionalización docente. En I. Cortés y C. Hirmas (eds.), Primer seminario sobre formación práctica docente: vinculación entre el sistema universitario y el sistema escolar (pp. 23-38). Chile.

De Zubiría, J. (2019, 17 de junio). ¿Cuál es el secreto de los grandes maestros? Semana. https://www.semana.com/opinion/articulo/cuales-el-secreto-de-los-grandes-maestros-columna-de-julian-de-zubiriasamper/620031

Eirín-Nemiña, R. (2018). Las comunidades de aprendizaje como estrategia de desarrollo profesional de docentes de Educación física. Estudios pedagógicos (Valdivia), 44(1), 259-278. https://dx.doi.org/10.4067/ S0718-07052018000100259

Espinoza, E. (2018). El tutor en los entornos virtuales de aprendizaje. Universidad y Sociedad, 10(3), 201-210.

Esquea, O. (2017). Sentidos de la práctica pedagógica en la formación docente. Caso Facultad de Educación - Universidad del Atlántico. Praxis, 13(2), 171-180. http://dx.doi.org/10.21676/23897856.2359

Font, V. (2011). Competencias profesionales en la formación inicial de profesores de matemáticas de secundaria. Revista Iberoamericana de Educación Matemática, Unión, (26), 9-25. 
Font, V. y Morales, Y. (2017). Análisis de la reflexión presente en las crónicas de estudiantes en formación inicial en Educación Matemática durante su periodo de práctica profesional. Revista de Ensino de ciências e Matématica, 122-137.

Fuentealba, A. y Vanegas, C. (2019). Identidad profesional docente, reflexión y práctica pedagógica: consideraciones claves para la formación de profesores. Perspectiva Educacional: formación de Profesores, 58(1), 115-138. http://dx.doi.org/10.4151/07189729-Vol.58-Iss.1-Art.780

González, K., Berdugo, C. N. y Mortigo, A. (2017). Incidencia de los entornos virtuales de aprendizaje en la calidad de la educación superior, desde el contexto colombiano. Revista Interamericana de Investigación, Educación y Pedagogía (RIIEP), 10(2), 11-24. https://doi.org/10.15332/ s1657-107X.2017.0002.01

Glasserma, L., Rubio, M. y Ramírez, M. (2013). Recursos educativos abiertos en la práctica docente. Virtualis, 4(8), 46-64.

Grajales, J. y Osorio, Y. (2019). La globalización y la importancia de las TIC en el desarrollo social. Revista reflexiones y saberes, 11, 2-9.

Hernández, M., Quezada, A. y Venegas, M. (2016). Análisis de la práctica docente en la formación inicial de profesores de religión. Educación y Educadores, 19(3), 357-369. http://dx.doi.org/10.5294/edu.2016.19.3.3

Hirmas, C. y Cortés, I. (2014). Conclusiones, tensiones y olvidos en la formación práctica docente. En I. Cortés y C. Hirmas (eds.), Primer seminario sobre formación práctica docente: vinculación entre el sistema universitario y el sistema escolar (pp. 8-21). Chile.

Instituto Colombiano para el Fomento de la Educación Superior (Icfes). (2018a). Marco de referencia para la evaluación Icfes. Ciencias de la Educación. Bogotá.

Instituto Colombiano para la Evaluación de la Educación (Icfes). (2018b). Saber Pro Informe Nacional de Resultados 2016-2017. Bogotá. https:// www.icfes.gov.co/documents/20143/238004/informe\%20nacional\%20 de\%20resultados\%20saber\%20pro\%202016\%202017.pdf 
Instituto Colombiano para la Evaluación de la Educación (Icfes). (2019). Instituciones de Educación Superior, reporte histórico de resultados del programa académico; Guía de interpretación y uso de resultados del examen Saber Pro. Bogóta.

Llinares, S., Ivars, P., Buforn, Á. y Groenwald, C. (2019). "Mirar profesionalmente" las situaciones de enseñanza: una competencia basada en el conocimiento. En E. Badillo, N. Climent, C. Fernández y M. González (eds.), Investigación sobre el profesor de matemáticas: práctica de aula, conocimiento, competencia y desarrollo propesional (pp. 177-192). Ediciones Universidad Salamanca.

Ministerio de Educación Nacional (MEN). (2006). Documento $n .^{\circ} 3$. Estándares Básicos de Competencia en Lenguaje, Matemáticas, Ciencias y Ciudadanas. Bogotá. https://www.mineducacion.gov.co/1621/articles340021_recurso_1.pdf

Ministerio de Educación Nacional (MEN). (2011). Perfil de competencias de directivos docentes y docentes. Bogotá.

Ministerio de Educación Nacional (MEN). (2009). Educación virtual o educación en línea. Bogotá. https://www.mineducacion.gov.co/1621/ article-196492.html

Ministerio de Educación Nacional (MEN). (2014). Dirección de Calidad para la Educación Preescolar, Básica y Media Subdirección de Referentes y Evaluación de la Calidad Educativa. Bogotá. https://www.mineducacion. gov.co/1759/articles-342767_recurso_3.pdf

Ministerio de Educación Nacional (MEN). (2016). La práctica pedagógica como escenario de aprendizaje. Bogotá. https://www.mineducacion. gov.co/1759/articles-357388_recurso_1.pdf

Ministerio de Educación Nacional. (2017, 15 de septiembre). Resolución 18583 de 2017. Por la cual se ajustan las características específicas de calidad de los programas de Licenciatura para la obtención, renovación o modificación del registro calificado, y se deroga la Resolución 2041 de 2016. Diario oficial 50357. 
Moreno, G. (2015). Formación inicial de docentes en metodología a distancia en Colombia. Aletheia, 7(2), 114-129.

Organización de las Naciones Unidas para la Educación, Ciencia y la Cultura (Unesco). (2013). Antecedentes y criterios para la elaboración de políticas docentes en en Ámerica Latina y el Caribe. https://unesdoc. unesco.org/ark:/48223/pf0000223249?fbclid=IwAR0zOMidNAWW8y4N bZG8FOaU2Nzjko7owuJucCNEOsta4_53k1bHjwTU9mU

Osorio, J., Herrera, L. y Quiles, O. (2016). Competencias docentes de los profesores en formación según opinión de los docentes formadores. Diá-logos, (17), 35-51. https://doi.org/10.5377/dialogos.v0i17.2740

Parra, J. (2013). La Práctica Educativa Bajo los Sistemas de Educación Superior a Distancia y Virtual. En N. Arboleda y C. Rama (eds.), La Educación Superior a Distancia y Virtual en Colombia: Nuevas Realidades, (175-184). Hipertextos Ltda.

Pedró, F. (2020). ¿Ofrecen las universidades una formación inicial docente suficientemente profesionalizadora? Revista Educación Superior y Sociedad (ESS), 32(1), 60-88.

Pino, L., Guzman, I., Larraín, M. y Vargas, C. (2018). La formación inicial de profesores en Chile: 'Voces' de la comunidad chilena de investigación en educación matemática. Uniciencia, 32(1), 68-88. http://dx.doi. org/10.15359/ru.32-1.5

Rico, L. (2013). El Método del Análisis Didáctico. Revista Iberoamericana de Educación Matemática, 33, 11-27

Rivero, E., Carmenate, L. y León, G. (2019). La profesionalización docente desde sus competencias esenciales. Experiencias y proyecciones del perfeccionamiento académico de la Universidad Técnica de Machala. Conrado, 15(67), 170-176. http://conrado.ucf.edu.cu/index.php/

Ruffinelli, A. (2013). La calidad de la formación inicial docente en Chile: la perspectiva de los profesores principiantes. Calidad en la Educación, (39), 118-153. http://dx.doi.org/10.4067/S0718-45652013000200005 
Schulmeyer, A. (2004). Estado actual de la evaluación docente en trece países de América Latina. En M. Vailant, T. Rego, B. Ávalos, G. Namo de Mello, E. Chezzi Dallan, G. Rama, J. Navarro, X. Liang, C. Herrán, C. Uribe, A. Mizala y P. Romaguera (eds.), Maestros en América Latina: Nuevas Perspectivas sobre su Formación y Desempeño (pp. 25-64). San Marino. https://www.thedialogue.org/wp-content/uploads/2016/03/2004Maestros-en-America-Latina-Nuevas-Perspectivas-sobre-su-Formaciony-Desempeno.pdf

Silva, J. (2010). El rol del tutor en los entornos virtuales de aprendizaje. Innovación Educativa, 10(52), 13-23.

Stake, R. E. (2006). Multiple Case Study Analysis. Guilford Publications.

Tejada, J. y Ruiz, C. (2016). Evaluación de competencias profesionales en Educación Superior: retos e implicaciones. Educación XX1, 19(1), 17-38. http://dx.doi.org/10.5944/educXX1.12175

Tobón, S. (2006). Aspectos básicos de la formación badasa en competencias. Proyecto Mesesup, Talca.

Tuning América Latina. (2007). En P. Beneitone, C. Esquetini, J. González, M. Maletá, G. Siufi y R. Wagenaar (eds.), Reflexiones y perspectivas de la Educación Superior en América Latina (pp. 33-70). Publicaciones de la Universidad de Deusto.

Vasilachis, I. (2006). Estrategias de Investigación Cualitativa. Editorial Gedisa, S.A. 